\title{
COMPARISON OF VARIOUS TISSUE AND CELL THERAPY APPROACHES WHEN RESTORING OVARIAN, HEPATIC AND KIDNEY'S FUNCTION AFTER CHEMOTHERAPY-INDUCED OVARIAN FAILURE
}

\author{
M.M. Kozub ${ }^{1}$, V.Y. Prokopiuk, *, K.P. Skibina ${ }^{3}$, O.V. Prokopiuk ${ }^{1}$, N.I. Kozub \\ ${ }^{1}$ Kharkiv Medical Academy of Post-Graduate Education, Kharkiv 61018, Ukraine \\ ${ }^{2}$ Institute for Problems of Cryobiology and Cryomedicine, NAS of Ukraine, Kharkiv 61002, Ukraine \\ ${ }^{3}$ Kharkiv National Medical University, Kharkiv 61145, Ukraine
}

\begin{abstract}
About $1 \%$ of women suffer from premature ovarian failure, which leads to a significant deterioration in the life quality. Most often this condition is caused by performed chemotherapy, autoimmune diseases, surgery performed on ovaries, uterus, or fallopian tubes. The aim of this study was to compare different approaches of tissue and cell therapy in restoring the sexual function in case of ovarian failure induced by chemotherapy. Materials and Methods: The study was carried out in BALB/c mice with the modeled ovarian failure, induced by cyclophosphamide and busulfan. The restoration dynamics of ovarian and sexual function, liver and kidneys after application of cryopreserved explants, cryoextract, placental mesenchymal stem cells, those of adipose tissue has been studied. Results: It has been shown that the use of various methods of cell and tissue therapy has comparable efficacy when treating an ovarian failure induced by chemotherapy. The most rapid and complete restoration of the reproductive system, liver and kidneys was observed when using the placental explants, extract and cells, but not with the use of mesenchymal stem cells of adipose tissue. No recovery of fertility in this experiment was observed. Conclusion: Various methods of cellular and tissue therapy are perspective in treatment of the chemotherapy complications. More effective are placental derivates.
\end{abstract}

Key Words: premature ovarian failure, chemotherapy, MSCs, placenta, menopause.

In developed countries, the physiological age of the menopause onset is 50-52 years. Premature ovarian failure (POF) is observed in $1 \%$ of women under 40 and $0.1 \%$ under 30 years, among those $37 \%$ is due to iatrogenic causes (mainly after oncology treatment), $25 \%$ - to idiopathic, $19 \%$ - to genetic, $19 \%$ to autoimmune $[1,2]$. Complications of premature menopause are not only the lack of fertility, decreased libido and amenorrhea, but also a sharp deterioration in the quality of life, psycho-emotional disorders, osteoporosis, increased mortality from cardiovascular diseases and strokes [3, 4]. Traditional hormone therapy requires constant drug intake, has a number of limitations, does not restore fertility and does not correct pathological changes in target organs [5]. Cell therapy, protection of the ovarian reserve by the administration of gonadotropin-releasing agonists prior to the chemotherapy, cryopreservation of ovarian tissue, gametes, embryos are referred to perspective methods of POF correction [6, 7].

The most curative and numerous groups of the patients with POF are the women with iatrogenic and autoimmune origin of the disease [1, 3, 8]. It should be noted that the use of methods of cell and tissue therapy, unambiguously shown in autoimmune diseases, is controversial in the patients with oncologic pathology. Along with the classical postulate, the worsening of cancer prognosis with the use of stimulants

Submitted: 24 April, 2017.

*Correspondence: E-mail: v.yu.prokopiuk@gmail.com Tel.: +380506159027

Abbreviations used: MSCs - mesenchymal stem cells; PBS phosphate buffered saline; POF - premature ovarian failure. and during pregnancy, there are the data on the ability of stem cells to suppress tumor growth independently and as a "vector" [11-14] as well as about the differentiated approaches to the restoration of fertility in the women who underwent oncology $[15,16]$. Most researchers use the introduction of various stem cells, often of either placental or adipose origin directly into the ovary, to restore the function of the ovaries that is effective in improving sexual function and fertility [17-20], but requires surgery, affects less other organs, demands the use of non-standardized culture techniques.

The research aim was to compare the different approaches of tissue and cell therapy when restoring chemotherapy-induced ovarian failure.

\section{MATERIALS AND METHODS}

In the research we used 90 female 6 -months old BALB/c mice weighing $20.2 \pm 0.4 \mathrm{~g}$ with a regular estrous cycle, which were divided into 6 groups of 15 animals each: 1 - the animals with modeled POF with no treatment, 2 - the animals with modeled POF and treatment with cryopreserved placental explants, 3 - the animals with modeled POF and treatment with placental cryoextract, $4-$ the animals with modeled POF and treatment with cryopreserved placental mesenchymal stem cells (MSCs), 5 - the animals with modeled POF and treatment with cryopreserved adipose tissue MSCs, 6 - intact animals.

POF was simulated according to the standard method [20] by means of administration of cyclophosphamide at a dose of $200 \mathrm{mg} / \mathrm{kg}$ (Baxter Oncology, Germany) and busulfan (Aspen Pharma, Germany) at a dose of $20 \mathrm{mg} / \mathrm{kg}$. Cyclophosphamide (200 mg) 
was dissolved in $20 \mathrm{ml}$ of phosphate buffered saline (PBS), busulfan $(20 \mathrm{mg}$ ) dissolved in $2 \mathrm{ml}$ of dimethylsulfoxide was added, and intraperitoneally administered by $0.5 \mathrm{ml}$ to each animal.

Placental explants were obtained from the human placenta by fragmentation of the villi as reported previously [21]. The placental extract was obtained by means of previously described method [22]. With this aim the human placenta delivered within $3 \mathrm{hr}$ after the cesarean section was fragmented, two parts of PBS were added to one part of the placental tissue, three times cooled by immersion in liquid nitrogen and warmed in a water bath at $37^{\circ} \mathrm{C}$, centrifuged at $1500 \mathrm{rpm} / \mathrm{min}$, the supernatant was removed and used. The placental MSCs were obtained by the method previously described, wherein the placenta cells were obtained by enzymatic method using $0.25 \%$ trypsin [23]. The cells were phenotyped earlier, CD90, CD73, CD105 markers were present on their surface, CD34 was absent, they had the ability to differentiate into osteogenic, adipogenic and chondrogenic directions [23]. MSCs of adipose tissue were obtained by the method [18] using enzymatic treatment with collagenase.

For a number of reasons, the cell therapy necessitates the use of cryopreserved objects [22]. Placental explants and MSCs were cryopreserved according to the previously used protocol [23], the DMEM with a high glucose content and L-glulamine (BioWest, France) enriched with $10 \%$ fetal bovine serum (Lonza, Germany) and 10\% dimethylsulfoxide (Sigma, USA) was used as the cryoprotective medium, were frozen in Nunc cryotubes (USA) using isopropanol containers. Mr. Frosty ${ }^{\mathrm{TM}}$ Freezing Container (Thermo Fisher Scientific, USA) at a rate of $1{ }^{\circ} \mathrm{C}$ down to $-70{ }^{\circ} \mathrm{C}$, followed by an immersion into liquid nitrogen. Thawing was performed in a water bath at $37^{\circ} \mathrm{C}$.

Treatment started 2 weeks later the chemotherapy. Cryopreserved explants of the placenta were intramuscularly injected by $10 \mathrm{mg}$ through a thick needle. The placenta extract was intramuscularly injected by $0.01 \mathrm{ml}$ once a day for 5 days. MSCs were intraperitoneally introduced once at $1 \cdot 10^{5}$ /animal. The dose and mode of administration were used in accordance with the recommendations for drug testing and publications [18, 20, 24].

The dynamics of changes in the weight of animals was studied. The function of the ovaries was examined by the method of vaginal cytology. The sexual function was examined by counting the number of vaginal plugs. Four weeks after the chemotherapy, 5 animals from each group were removed from the experiment, the preparations of ovaries, the uteri as organs of reproductive system, liver and kidneys as detoxification organs were histologically examined. After 12 weeks, all the animals were removed from the experiments.

During the experiment, the animals were exposed to natural light, they received standard food ad libitum. To assess sexual and reproductive functions, females were kept with 6-months old intact BALB/c males at 1:3 ratio. The maintenance of animals corresponded to the current rules on equipping and keeping the vivarium.

The results were statistically processed by the ANOVA method using Past V. 3.15 (Hammer, Natural History Museum, University of Oslo) software.

The placentas were obtained with the informed consent of women after the cesarean section surgery. The experiments were carried out in accordance with the "General Principles of the Experiments in Animals", approved by the $5^{\text {th }}$ Congress in Bioethics (Kyiv, 2013) and coordinated with the statements of the "European Convention for the Protection of Vertebrate Animals Used for Experimental and Other Scientific Purposes" (Strasbourg, 1986), agreed with the Committee in Bioethics of the Institute for Problems of Cryobiology and Cryomedicine of the National Academy of Sciences of Ukraine (Protocol No. 2 of June $3^{\text {rd }}$, 2013).

\section{RESULTS AND DISCUSSION}

During studying the dynamics of animals' weight for 8 weeks, the body mass of mice of the control group increased from 19.8 up to $22.2 \mathrm{~g}$, being the feature of mice of this age. In all the groups after chemotherapy, the weight dropped sharply down to $17.5 \mathrm{~g}$ followed by a slow recovery. Simultaneously, at the same time, we found a change in their general condition, i.e. hypodynamia, felted hair, eye opacity. In the group with no treatment, the mice were restored to the intact group for 8 weeks. The group injected with the placenta explants restored the weight for 5 weeks after chemotherapy, the group that received the placental cryoextract did for 6 weeks, and the groups did after the introduction of the cells for 7 weeks (Fig. 1, a). Simultaneously with the restoration of weight, the general condition and appearance of the animals improved.

When investigating the number of estrous cycles by the method of vaginal cytology in the control group, a regular cycle was observed in all the animals. In the animals after chemotherapy, the absence of superficial epithelium was noted, it began to appear 5 weeks after chemotherapy, and after 8 weeks it was observed in $50 \%$ of mice that was in accordance with the reports on this model [20]. In the group after the introduction of placental explants, the cyclicity was restored quickly, and 6 weeks after chemotherapy it was observed in 100\% of the animals. The use of placental cryoextract restored the estrous cycle in $80 \%$ of the animals to the $5^{\text {th }}$ week. The use of cells made it possible to achieve a result to the week $7-8$ in $70 \%$ of animals (Fig. 1, b). It should be noted that after chemotherapy in most animals, a regular 4-day estrous cycle was not restored, there was a periodic appearance of a superficial epithelium in the smear of keratinized cells for a period from 3 to 8 days, which indicated the estrogen saturation of the organism, possible without ovulation. Restoration of the estrous cycle was correlated with the weight of mice, so when comparing the weights of the animals and the onset of regular estrous cycle, 

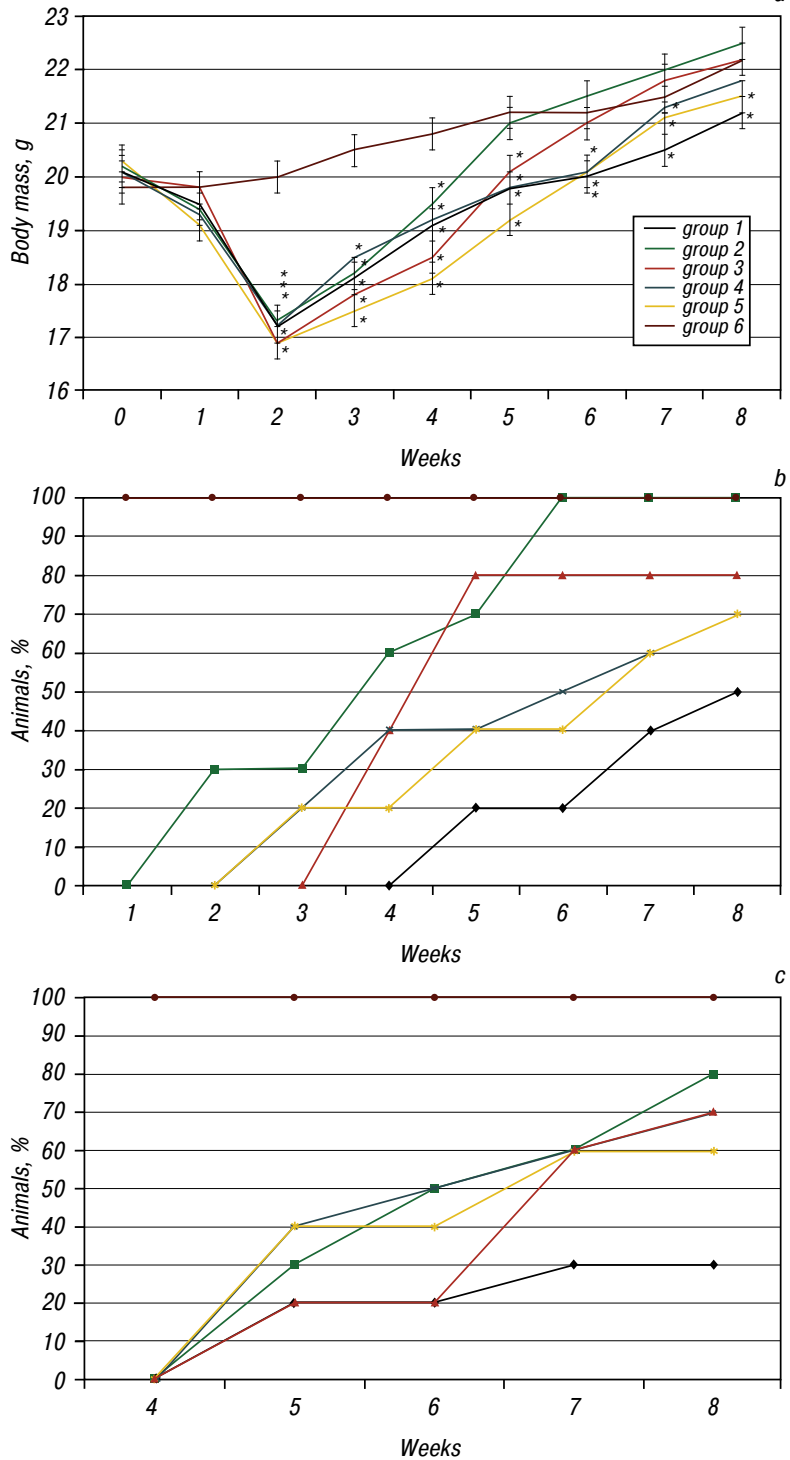

Fig.1. Dynamics of changes in body mass of animals (a), recovery of estrous cycles $(b)$ and sexual activity $(c)$, mean \pm SD. ${ }^{\star} p<0.05$ (compared with control)

it was noted that mice weighing less than $18 \mathrm{~g}$ rarely had a regular estrous cycle.

When studying the sexual function it has been shown that the number of effective couplings in the group after chemotherapy made $30 \%$ at week 8 that corresponded to the published data for the model
(Fig. 1, c). With the use of various methods of therapy, this index increased up to $60-80 \%$, higher when the explants were used, less if using the placental cryoextract and cells were applied.

In the investigation of reproductive function $90 \%$ of the control group females became pregnant during the experiment, an average number of fetuses was 12 . None of the females after the chemotherapy became pregnant. According to the literature data for this model, restoration of fertility was described in the case when the cells were introduced directly into the ovary [20].

When the animals were removed from the experiment at week 4 in intact animals the ovaries were of the usual size with follicles of various maturity (Fig. 2, a), all the layers and glands were visible in the uterus (Fig. 2, b), the kidneys were of usual structure with preserved glomerular and tubular apparatus (Fig. 2, c), the liver with preserved lobular structure (Fig. 2, d). In the animals after chemotherapy, the decreased size of uteri and ovaries has attracted the attention. In histological examination a reduced number of primary follicles, complete absence of secondary ones, sharp atrophy, and shrinkage cells and organ as a whole were found in ovaries (Fig. 2, e). In uterus a strong atrophy with thinning of all the layers was observed (Fig. 2, f). Changes in the reproductive system did not differ in all the groups receiving chemotherapy. In the kidneys a sharp edema of the cortical and medular layers was observed (Fig. 2, g), however, in the groups using the placental explants and cryoextract only a part of the kidney was edematous, not more than a half of the sections' area (Fig. $2, h$ ). In the study of liver, edema and its impaired lobular structure were observed in all the groups (Fig. 2, i), however, in the groups treated with cryoextract and placenta explants, the lobular structure was partially preserved (Fig. $2, j$ ).

After 12 weeks when animals were removed from the experiment in the group after chemotherapy without treatment, a sharp increase in adipose tissue in the abdominal cavity was observed. The ovaries were reduced in size, histological examination indicated the absence of structural elements in them: yellow bodies, primordial, primary, secondary follicles, the stroma was filled with large cells (Fig. 3,a). The restoration of estrogen saturation of the body in this
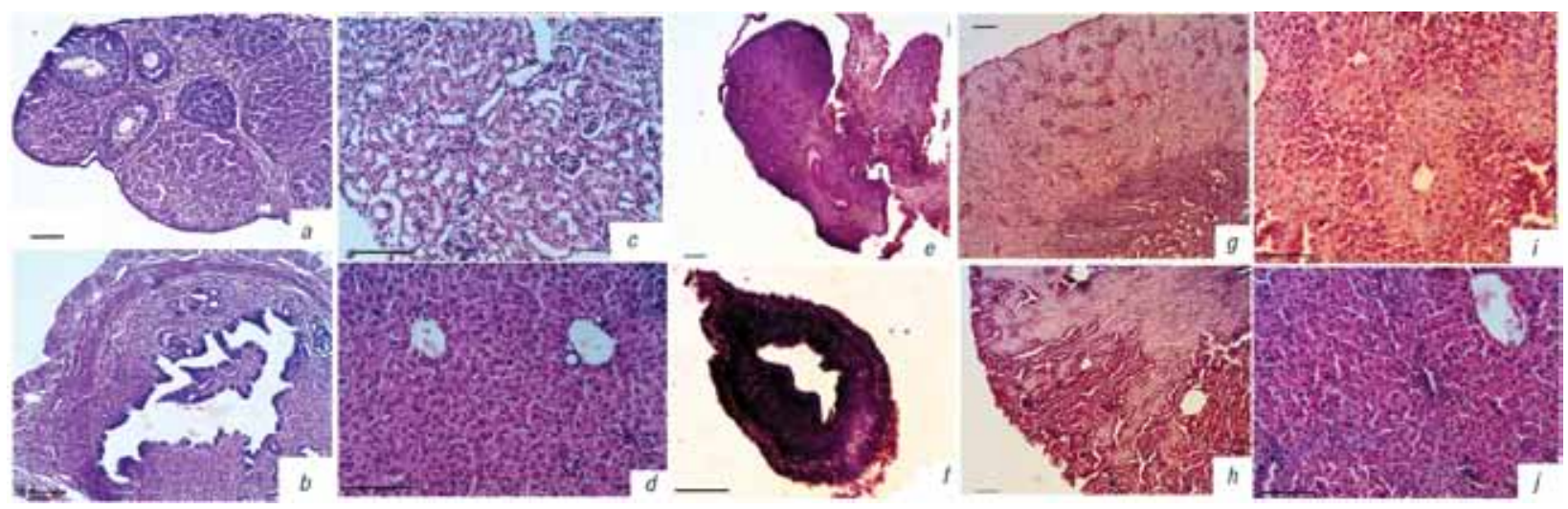

Fig. 2. Morphological changes in organs of studied animals in 4 weeks after chemotherapy: $a-d-$ control group, $e-j-$ experimental groups. Hematoxylin-eosin, scale bar $100 \mu \mathrm{m}$ 
group of animals was evidently due to an adipose tissue, but not ovaries. Histological examination of uteri showed less pronounced atrophic events than at week 4 after chemotherapy, but the thinning of layers as well as endometrium and myometrium atrophy was significant, the lumen was enlarged, the number of glands decreased (Fig. $3, b$ ). Histological examination of kidneys demonstrated the shrinkage of the glomeruli and a persistent edema of tubules (Fig. 3, c). In liver the foci of cirrhosis, disordered lobular structure, and areas of nuclear polymorphism were observed (Fig. 3, d).

In the study of the ovaries of animals treated with placental explants the structural elements were also absent, but the follicle-like formations, i.e. the rounded areas containing large cells with a light cytoplasm (Fig. 3, e) were also enlarged if compared with the previous group. Histological examination of uteri showed an increase in the number of glands, endometrial hyperplasia, myometrium thickening (Fig. 3, f), organ structure differed slightly from intact animals. In kidneys the minimal changes in glomerular and tubular apparatus were observed (Fig. 3, g). In liver the lobular structure was preserved, there were no cirrhosis foci, however polymorphism of nuclei was found (Fig. $3, h$ ).

In animals treated with placental cryoextract in the ovaries, there were no typical elements, individual follicle-like structures were observed (Fig. 3, i) with the size of the ovaries smaller than in the previous group. Uterus was close to the intact (Fig. $3, j$ ), liver was with preserved lobular structure, in some cases we found the cell polymorphism, heterogeneity. The kidneys had the preserved structure, there was a shrinkage of individual glomeruli.

When investigating the groups treated with MSCs of both a placental (Fig. 3, $k, I$ ) and adipose origins (Fig. 3, $m, n$ ), the structure of organs differed slightly. In both groups there were no typical elements in ovaries, the hyperplasia of either individual cells or cell groups was noted. The structure of uteri was close to native, with some atrophy of the layers, the number and size of glands were increased. In kidneys the structure was preserved, there was a shrinkage of some glomeruli. In liver we noted the preserved lobular structure, individual sites of accumulation of small nuclei.

In addition, it should be noted that a response of animals to chemotherapy and treatment was different. So in the first group in the ovaries of one animal the folliculogenesis was preserved, but not there was no formation of yellow bodies and 12 weeks later no changes in the liver and kidneys. In one animal of the second group in response to the introduced explants a sharp endometrial hyperplasia was observed, with
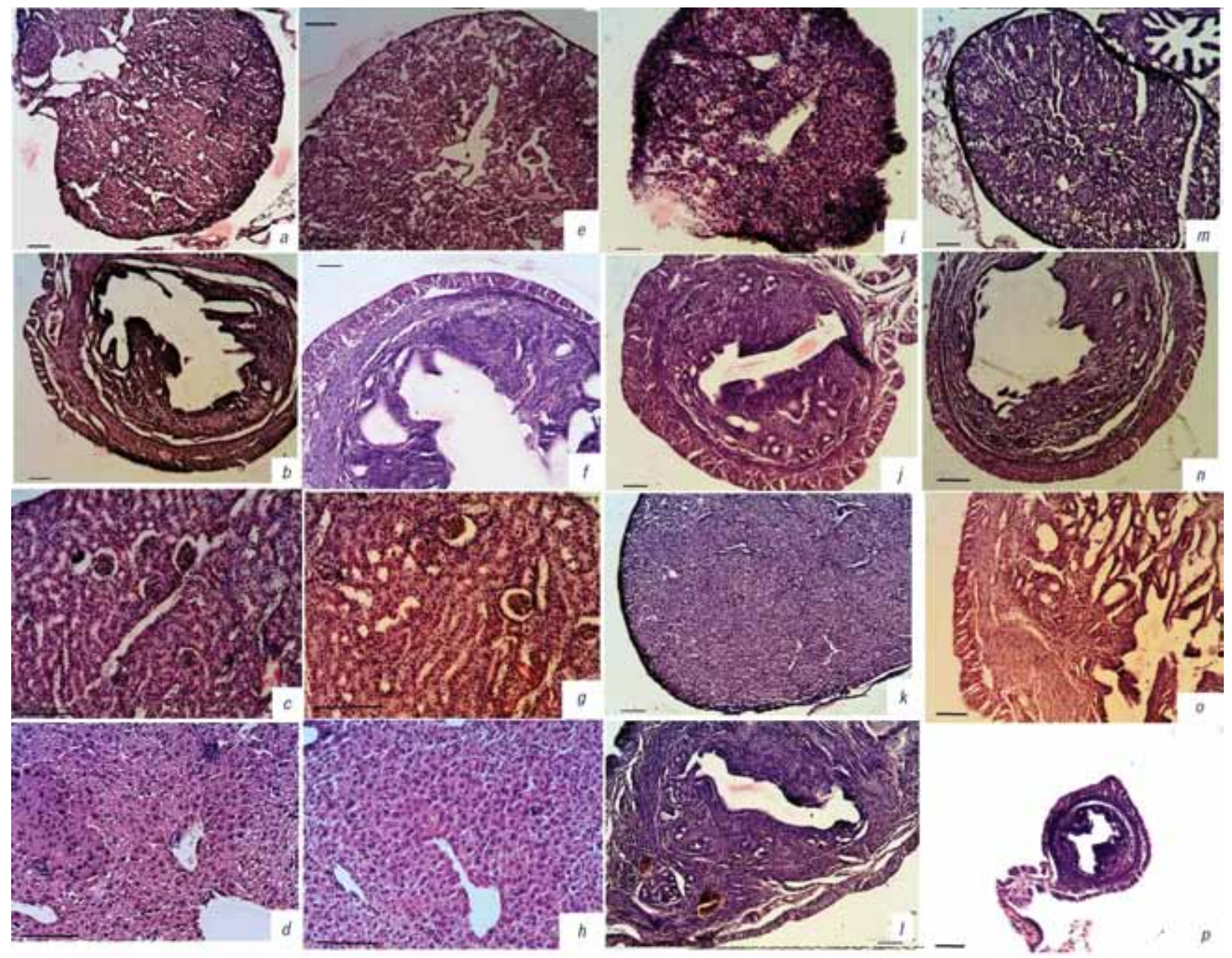

Fig. 3. Morphological changes in organs of studied animals in 12 weeks after chemotherapy: $a-d-$ group 1 ; $e-h, o-$ group 2 ; $i-j, p-$ group $3 ; k, I$-group $4 ; m, n$ - group 5 . Hematoxylin-eosin, scale bar $100 \mu \mathrm{m}$ 
complete ovarian atrophy (Fig. $3, o$ ). One animal of the third group demonstrated a severe atrophy of the uterus at week 12 (Fig. 3, p), cirrhotic changes in the liver, despite gaining the weight. However, the changes in the remaining animals in the groups were of the same type.

The performed studies enabled to draw the following conclusions: the use of various methods of cell and tissue therapy (placental explants, cryoextract, cells) had a comparable efficacy when treating the ovarian failure, induced by chemotherapy. With systemic administration of drugs (non-topical), it is possible to restore kidneys, liver, ovarian function, but not fertility. The most rapid and complete restoration of reproductive system, liver and kidneys was observed when using the organotypic culture and cryoextract of placenta. The use of MSCs derived from various sources led to a slower recovery. Sensitivity to chemotherapy and treatment in individuals depended on the specific characteristics of an organism.

\section{FUNDING SOURCES}

The research was carried out within the framework of the R\&D topic Ref. № 2.2.6.89 "Investigation of geroprotective and gerotherapeutic effect of placental bioobjects", R\&D № 0113 U002955 "Genetic modification and long-term storage of the placental stem cells for clinical use".

\section{ACKNOWLEDGMENTS}

The authors are grateful to the employees of the Medical University of Hannover, Germany (Hannover Medical School, Germany) Dr. Thomas Mueller, Dr. Denys Pogozhykh, Dr. Olena Pogozhykh for assistance in performing the research (advisory assistance when working with placental MSCs).

\section{CONFLICT OF INTEREST}

The authors state that there are no conflicts of interest in this study.

\section{REFERENCES}

1. Fenton AJ. Premature ovarian insufficiency: Pathogenesis and management. J Midlife Health 2015; 6: 147-53.

2. Panay N, Fenton A. Premature ovarian insufficiency: Working towards an international database. Climacteric 2012; 15: 295-6.

3. Liem GS, Mo FK, Pang E, et al. Chemotherapy-related amenorrhea and menopause in young chinese breast cancer patients: analysis on incidence, risk factors and serum hormone profiles. PLoS One 2015; 10: e0140842.

4. Podfigurna-Stopa A, Czyzyk A, Grymowicz M, et al. Premature ovarian insufficiency: the context of long-term effects. J Endocrinol Invest 2016; 39: 983-90.

5. Sullivan SD, Sarrel PM, Nelson LM. Hormone replacement therapy in young women with primary ovarian insufficiency and early menopause. Fertil Steril 2016; 106: 1588-99.

6. Blumenfeld $Z$. How to preserve fertility in young women exposed to chemotherapy. The role of GnRH agonist cotreat- ment in addition to cryopreservation of embrya, oocytes, or ovaries? Oncologist 2007; 12: 1044-54.

7. Gerber B, Ortmann O. Prevention of early menopause study (POEMS): Is it possible to preserve ovarian function by gonadotropin releasing hormone analogs (GnRHa)? Arch Gynecol Obstet 2014; 290: 1051-3.

8. Ayesha JV, Goswami D. Premature ovarian failure: an association with autoimmune diseases. J Clin Diagn Res 2016; 10: 10-2.

9. Jeon SJ, Lee JI, Jeon MJ, Lee M. Prognostic effects of adjuvant chemotherapy-induced amenorrhea and subsequent resumption of menstruation for premenopausal breast cancer patients. Medicine (Baltimore) 2016; 95: e3301.

10. Radecka B, Litwiniuk M. Breast cancer in young women. Ginekol Pol 2016; 87: 659-63.

11. Li Z, Fan D, Xiong D. Mesenchymal stem cells as delivery vectors for anti-tumor therapy. Stem Cell Investig 2015; $2: 6$.

12. Sage EK, Thakrar RM, Janes SM. Genetically modified mesenchymal stromal cells in cancer therapy. Cytotherapy 2016; 18: 1435-45.

13. Uchibori $R$, Tsukahara $T$, Ohmine $K$, et al. Cancer gene therapy using mesenchymal stem cells. Int $\mathbf{J}$ Hematol 2014; 99: 377-82.

14. Silini AR, Cancelli S, Signoroni PB, et al. The dichotomy of placenta-derived cells in cancer growth. Placenta 2017; DOI: 10.1016/j.placenta.2017.05.011.

15. Córdoba O, Bellet M, Vidal X, et al. Pregnancy after treatment of breast cancer in young women does not adversely affect the prognosis. Breast 2012; 21: 272-5.

16. Margulies AL, Selleret L, Zilberman S, et al. Pregnancy after cancer: for whom and when? Bull Cancer 2015; 102: 463-9.

17. Lai D, Wang F, Yao X. Human endometrial mesenchymal stem cells restore ovarian function through improving the renewal of germline stem cells in a mouse model of premature ovarian failure. J Transl Med 2015; 13: 155.

18. Sun M, Wang S, Li Y, et al. Adipose-derived stem cells improved mouse ovary function after chemotherapy-induced ovary failure. Stem Cell Res Ther 2013; 4: 80.

19. Edessy M, Hosni Hala N, Shady Y, et al. Autologous stem cells therapy, the first baby of idiopathic premature ovarian failure. Acta Medica Int 2016; 3: 19-23.

20. Xiao GY, Liu IH, Cheng CC, et al. Amniotic fluid stem cells prevent follicle atresia and rescue fertility of mice with premature ovarian failure induced by chemotherapy. PLoS One 2014; 9: e106538.

21. Prokopyuk VYu, Prokopyuk OS, Musatova IB, et al. Safety of placental, umbilical cord and fetal membrane explants after cryopreservation. Cell Organ Transplantol 2015; (1): 34-8.

22. Schevchenko NO, Somova KV, Volina VV, et al. Dynamics of activity and duration of functioning of cryopreserved cryoextract, placental cells and fragments in the organism of experimental animals. Morphologia 2016; 10: 93-8.

23. Pogozhykh D, Prokopyuk V, Pogozhykh O, et al. Influence of factors of cryopreservation and hypothermic storage on survival and functional parameters of multipotent stromal cells of placental origin. PLoS One 2015; 10: 1-25.

24. Preclinical trials of medicines [guidelines]. Stefanov OV, ed. Kyiv: Avitsena, 2001. 528 p. (in Russian). 